Revue d'Allemagne et des pays de langue allemande

50-2 | 2018

Humanités environnementales - Quoi de neuf du côté des méthodes?

\title{
La notion de « race juive » sous la plume d'Arthur Ruppin (1876-1943)
}

Jérôme Mancassola

\section{(2) OpenEdition}

Journals

Édition électronique

URL : https://journals.openedition.org/allemagne/985

DOI : 10.4000 /allemagne. 985

ISSN : 2605-7913

Éditeur

Société d'études allemandes

Édition imprimée

Date de publication : 30 décembre 2018

Pagination : 429-440

ISSN : 0035-0974

Référence électronique

Jérôme Mancassola, "La notion de « race juive » sous la plume d'Arthur Ruppin (1876-1943) », Revue d'Allemagne et des pays de langue allemande [En ligne], 50-2 | 2018, mis en ligne le 30 décembre 2019, consulté le 18 mai 2021. URL : http://journals.openedition.org/allemagne/985 ; DOI : https://doi.org/ 10.4000/allemagne.985 


\section{La notion de «race juive» sous la plume d'Arthur Ruppin $(1876-1943)^{(1)}$}

- Jérôme Mancassola*

«Il ne vaut pas la peine d'essayer de faire en sorte que I'histoire ne se répète pas, car le caractère de l'homme rendra toujours impossible la prévention des répétitions »(2).

Mark Twain

Juif et race: rares sont les termes qui auront autant repoussé les limites de la controverse intellectuelle. Leur définition protéiforme, leurs paradoxes troublants ainsi que la radicalité idéologique inouïe résultant de leur interaction en font un sujet d'étude passionnant; c'est un alliage terminologique non seulement ancien, mais aussi d'une durabilité historique déconcertante, lorsque l'on étudie dans le cadre de la longue histoire des idées deux nations incontournables: l'Allemagne et Israël.

Le mémoire dont est tiré cet article propose une analyse historique des interactions conceptuelles entre juif et race, en particulier celles qui peuvent être faussement perçues comme paradoxales. Pour saisir au mieux ces "paradoxes", gardons en tête le contexte européen des $\mathrm{XIX}^{\mathrm{e}}$ et $\mathrm{XX}^{\mathrm{e}}$ siècles. Personne ne méconnaît - ou n'irait contester sérieusement - l'essor de l'antisémitisme, du nationalisme, de l'eugénisme et du racisme en Europe, de la seconde moitié du XIX ${ }^{e}$ siècle jusqu'à la première moitié du $\mathrm{XX}^{\mathrm{e}}$ siècle. Les théories selon lesquelles les juifs appartiendraient biologiquement à une

* Doctorant contractuel en histoire au sein de l'École doctorale des humanités de l'Université de Strasbourg (ED 520).

1 Cet article, achevé en juin 2018, reprend les conclusions du mémoire éponyme: Jérôme MANCASsola, La notion de "race juive» sous la plume d'Arthur Ruppin (1876-1943), mémoire de master en histoire, Université de Strasbourg, Faculté des sciences historiques, 2017, 177 p., non publié.

2 Mark Twain, Mark Twain in Eruption: Hitherto Unpublished Pages About Men and Events, New York/ Londres, Bernard DeVoto, 1940: «It is not worth while to try to keep history from repeating itself, for man's character will always make the preventing of the repetitions impossible.» 
race humaine nuisible pour l'espèce ont bel et bien existé( ${ }^{(3)}$. Cependant, l'inverse aussi : durant cette même période, certains penseurs juifs étaient convaincus de l'existence d'une race juive - les griefs judéophobes en moins. Ce fait est susceptible de choquer les non avertis. Pour autant, il s'agit là d'un piège du regard rétrospectif où le terme «race» renvoie presque invariablement, dans l'imaginaire collectif, à la barbarie nazie. Si on s'extirpe de cette construction mentale, on se rend compte qu'il s'agit d'un faux paradoxe, dont les échos résonnent encore: les néonazis n’ont pas disparu en 2017, tout comme certains juifs qui se cherchent désespérément un ADN juif ${ }^{(4)}$.

Nos recherches avaient pour ambition première de se focaliser sur ces raciologues juifs des $\mathrm{XIX}^{\mathrm{e}}$ et $\mathrm{XX}^{\mathrm{e}}$ siècles. Premier constat: les travaux en la matière représentent peu de choses, si on les compare à l'abondante masse de recherches concernant les raciologues antisémites. Mais, depuis la seconde moitié des années 1990, la raciologie juive est devenue un champ de recherche en expansion, avec pour enjeux d'analyser et de synthétiser l'environnement culturel et les théories des raciologues ${ }^{(5)}$. Nous nous y sommes inscrit et focalisé sur un savant précis: le sioniste et sociologue allemand Arthur Ruppin.

Né en 1876 en Prusse dans la province de Posnanie, Arthur Ruppin fut un sioniste de premier plan. Envoyé en Palestine par la World Zionist Organization à la fin des années 1900, il y créa le Palestine Office - le centre administratif du Nouveau Yishouv ${ }^{(6)}$ - et acheta des terres pour le mouvement sioniste. Planificateur économique et foncier hors pair, il eut des activités d'une diversité impressionnante: il fut précurseur du kibboutz, défenseur de la politique d'installation sioniste à chaque commission d'enquête britannique, fondateur d'une cour de justice en 1909, promoteur d'une culture hébraïque moderne auprès des nouveaux arrivants... Mais en parallèle, Ruppin était aussi un universitaire. Diplômé en droit et considéré comme l'un des pères de la sociologie juive, il publia des travaux à la croisée de la sociologie, de la démographie et de l'anthropologie raciale. Après avoir pris en 1904 la direction du Büro für Statistik der Juden à Berlin, il constitua une base de données statistiques sans précédent sur les juifs du monde entier. Pour Ruppin, ces recherches avaient un but précis: établir un inventaire de la situation des juifs en Europe, s'en servir ensuite pour - selon lui - «corriger» leurs «faiblesses» biologiques et mentales; et enfin, régénérer de manière quasi-eugénique en Palestine une nouvelle race juive, fière et forte.

Étudier les théories d'Arthur Ruppin semblait plus pertinent que celles de tout autre raciologue. En dehors du fait qu'il fut l'un des rares à avoir tenté d'appliquer ses théories raciales en Palestine, il compila surtout les recherches antérieures de ses pairs,

3 Sur l'antisémitisme biologique et racial, cf. André Ріснот, La société pure: de Darwin à Hitler, Paris, Flammarion, 2009, p. 393-423.

4 Nous y reviendrons à la fin de l'article.

5 Parmi les ouvrages incontournables traitant de la raciologie juive, citons en particulier John M. Efron, Defenders of the Race: Jewish Doctors and Race Science in Fin-de-Siècle Europe, New Haven, Yale University Press, 1994; Mitchell Bryan HART, Social Science and the Politics of Modern Jewish Identity, Stanford (Californie), Stanford University Press, 2000; Derek Jonathan Penslar, Zionism and Technocracy: the Engineering of Jewish Settlement in Palestine, 1870-1918, Bloomington, 1991.

6 C'est-à-dire l'ensemble des populations juives qui ont immigré et se sont installées en Palestine avant la création de l'État d'Israël, entre 1882 et 1948. 
qu'il prolongea par sa réflexion propre, fortement influencée par les idées raciales allemandes de son temps. Enfin, il fut également un pionnier qui introduisit l'usage systématique de la sociologie et des statistiques dans les études raciales juives.

Nos travaux partent d'un deuxième constat: aucune synthèse de la pensée raciale «ruppinienne» n'avait encore été réalisée. Les travaux datant des années 1990 cités plus haut - et même les rares ouvrages et articles centrés sur Arthur Ruppin n'abordent ses théories raciales que de manière parcellaire ou insuffisante - au profit des domaines économique, politique et sociologique ${ }^{(7)}$. L'excellent ouvrage d'Etan Bloom ${ }^{(8)}$ fait exception, même s'il insiste plus sur la mise en pratique des théories ruppiniennes que sur les théories elles-mêmes.

À partir d'une relecture renouvelée et sélective des œuvres du sociologue ${ }^{(9)}-$ c'est- $^{\text {'e }}$ à-dire tournée sur les passages qui nous éclairent sur ses concepts théoriques plus que sur leur mise en pratique - ainsi que ses travaux universitaires précédemment cités, nos recherches avaient deux objectifs. Le premier était de proposer une synthèse de l'idéologie raciale d'Arthur Ruppin, dans le but d'en extraire une vue d'ensemble théorique inédite du courant sioniste racial allemand, irrigué par moult domaines connexes de l'époque - eugénisme, darwinisme social, sociologie, biologie... Par extension, il s'agissait de s'intéresser à un champ d'étude encore peu connu dans le monde francophone. En filigrane, le second objectif était de démystifier un corpus racial complexe et fantasmé, autrement dit de démontrer que l'on peut retracer la lente maturation historique de la notion de «race juive» au sein de certains groupes intellectuels juifs européens et par conséquent briser ce faux «paradoxe»: celui du développement concomitant de l'antisémitisme racial et de la raciologie juive. Nous verrons à la fin de cet article que ce paradoxe persiste jusqu'à nos jours.

Nous avons cherché à montrer en quoi la notion de " race juive » d'Arthur Ruppin est une synthèse théorique notoire du sionisme biologique, eugénique et sociologique allemand. L'analyse fut divisée en trois grandes parties. La première revient sur les facteurs idéologiques qui ont amené le sociologue à théoriser l'existence d'une race juive. Il s'agit là de poser le cadre idéologique et historique dans lequel il s'est ancré, tout en soulignant ses propres apports à la raciologie juive, sioniste et allemande. La deuxième partie présente sa vision historique et raciale - ou «bio-historique» - du peuple juif. Quant à la troisième, elle aborde son diagnostic des «déficits» socio-raciaux des juifs contemporains, ainsi que les solutions eugéniques qu'il promut. Ces deux dernières parties montrent à quel point le système racial ruppinien était un condensé de

7 Sergio Dellapergola, «Arthur Ruppin Revisited: The Jews of Today, 1904-1994», in: Steven M. Cohen, Gabriel Horencyzk, National Variations in Jewish Identity, 1999, State University of New York Press, http://www.bjpa.org/Publications/details.cfm?PublicationID=151; Yaakov Goren, Arthur Ruppin. His Life and his Work, Jerusalem, Yad Tabenkin; Amos MorRIs-ReICH, «Arthur Ruppin's Concept of Race», Israel Studies, 11/3 (2006), consulté le 18 avril 2016, http://img2.timg.co.il/ CommunaFiles/38845410.pdf

8 Etan Bloom, Arthur Ruppin and the Production of Pre-Israeli Culture, Boston, Brill, 2011.

9 En particulier quatre ouvrages: Arthur Ruppin, The Jews of Today, New York, Henry Holt and Company, 1913; Arthur Ruppin, Soziologie der Juden, Berlin, Jüdischer Verlag, 1930, 2 vol.; Arthur RupPIN, The Jewish Fate and Future, Westport (Connecticut), Greenwood Press, 1972; Arthur Ruppin, Alex Bein, Arthur Ruppin: Memoirs, Diaries, Letters, Londres, Weidenfeld and Nicolson, 1971. 
l'ensemble des thématiques qui irriguait le sionisme racial allemand - racialisation de l'histoire, usage des statistiques, attrait pour l'eugénisme...

\section{Genèse et mûrissement d'un « cocktail idéologique » original}

Il importait d'abord d'identifier les sources d'inspiration de la pensée ruppinienne. En examinant ses mémoires et son journal ${ }^{(10)}$, il fut possible de retracer l'évolution de sa réflexion sioniste et raciale, ainsi les quatre ingrédients qui constituèrent le «cocktail idéologique» ruppinien - c'est-à-dire l'origine du sionisme racial allemand - : l'antisémitisme, les sciences sociales et statistiques, les théories raciales et le sionisme pratique.

Toute la psychologie ruppinienne s'est forgée autour de l'antisémitisme allemand des années 1870 . Il a connu cette époque charnière où l'antijudaïsme chrétien ancien fut supplanté par une nouvelle judéophobie laïque: l'antisémitisme politique et racial. Dans toutes les sociétés occidentales en voie d'industrialisation et de sécularisation, de nouveaux partis politiques émergèrent, s'insurgèrent contre l'émancipation des juifs et en firent la seule et unique cause de tous les maux des sociétés modernes. En Allemagne, après le Gründerkrach de 1873 et les problèmes économiques qui en découlèrent, le juif devint l'archétype du bouc émissaire. De plus, après l'unification nationale de 1870, un décalage persistait entre la confiscation du pouvoir par les conservateurs prussiens et les aspirations démocratiques du peuple. Pour combler ce vide, un nationalisme «populaire» émergea, via des partis et des associations autonomes. Auto-radicalisés, ils combattaient toute opposition ou «corps étrangers» - tels que les juifs - susceptibles de remettre en cause l'union nationale. Dans sa forme tardive et exacerbée mais minoritaire - des années 1890, cette haine nationaliste, désormais impérialiste et «légitimée» par de pseudo-théories raciales, assimila le juif à un virus mortel, un élément exogène à expulser - ou à éliminer - si l'on souhaitait que le corps national allemand n'en meure pas ${ }^{(11)}$. Cette haine transparaissait abondamment dans les écrits de jeunesse de Ruppin. Ses années d'études et d'exercice dans la fonction publique ${ }^{(12)}$ furent marquées par la peur constante de l'avenir et le sentiment d'infériorité au sein de cette société chrétienne et nationaliste. Comme ses futurs collègues sionistes, cette impossibilité à nouer des relations d'égal à égal avec des non-juifs l'amena à douter de l'avenir du peuple juif en Allemagne et le poussa à s'intéresser au peuple juif ${ }^{(13)}$.

Au-delà de l'antisémitisme, le sionisme racial de Ruppin fut façonné par l'étude de la sociologie et des statistiques. À la fin du XIX ${ }^{\mathrm{e}}$ siècle, les sciences sociales allemandes cherchaient à analyser, de manière large, les faits sociétaux contemporains (à travers

10 A. Ruppin, Arthur Ruppin: Memoirs, Diaries, Letters (note 9).

11 En ce qui concerne l'antisémitisme et le nationalisme en Allemagne, cf. Dorothea BoHNEKAMP, De Weimar à Vichy: Les Juifs d'Allemagne en République, 1918-1940, Paris, Fayard, 2015, p. 117-121; JeanPaul Cahn, Bernard Poloni, Gérard Schneilin (dir.), Le Reich allemand du départ de Bismarck à la Première Guerre mondiale, 1890-1914, Nantes, Éditions du Temps, 2003, p. 166-177; Léon PoliAkov, Histoire de l'antisémitisme, tome 2: L'âge de la science, Paris, Seuil (coll. Points histoire), 1991, p. 211222, 272-283.

12 Diplômé en droit en 1902, il commença une carrière d'avocat, jusqu'à son départ définitif en Palestine, en 1907.

13 A. Ruppin, Arthur Ruppin: Memoirs, Diaries, Letters (note 9), p. 28, 50, 61-65. 
l'économie, la démographie, la sociologie, mais aussi la biologie, l'anthropologie, la médecine...) au détriment des explications historiques et religieuses classiques. La méthode, elle, se basait largement sur des études statistiques ${ }^{(14)}$. Ruppin était convaincu du pouvoir des nombres, de leur capacité à régler de grands problèmes sociétaux, tels que ceux de la communauté juive. En effet, selon l'historien américain H. Stuart Hugues, il appartenait à cette "génération 1890 », composée d'hommes brillants - tels que Max Weber ou Werner Sombart - balayant tous les systèmes de pensée (comme le marxisme ou le darwinisme social) et désireux de résoudre les grandes problématiques de la société moderne. Ainsi, dès 1903, il se mit à sillonner l'Europe de l'Est, dans le but de collecter sur place des informations pertinentes sur les communautés juives qui $y$ vivaient ${ }^{(15)}$. Certes, Ruppin ne fut pas le premier juif à réaliser de telles enquêtes de terrain sur ses coreligionnaires. Cependant, il égala largement les travaux antérieurs tels que ceux de Léopold Zunz et d'Alfred Nossig ${ }^{(16)}$ - en compilant, au sein du Bureau für Statistik der Juden, une quantité inédite de données statistiques sur les juifs du monde entier - qui furent d'ailleurs utilisées durant tout le $\mathrm{XX}^{\mathrm{e}}$ siècle ${ }^{(17)}$. Mais plus encore, il fut celui qui amena le mouvement sioniste racial à user systématiquement des statistiques comme méthode d'analyse sociologique et eugénique. La pensée raciale d'Arthur Ruppin était donc structurée par une méthode empirique et statistique.

Sa théorie raciale, à la croisée de la sociologie, de l'eugénisme et du darwinisme social, fut largement le fruit d'idées antérieures ${ }^{(18)}$. L'Allemagne fin-de-siècle d'Arthur Ruppin fut le théâtre d'un impressionnant télescopage idéologique: aryanisme de Gobineau, eugénisme radical - stérilisation, euthanasie... -, taxonomie, darwinisme social, hygiène raciale, nationalisme exacerbé... ${ }^{(19)}$. Tout comme ses collègues raciologues Joseph Jacobs, Elias Auerbach et Ignace Zollschan, Arthur Ruppin puisa à tous ces courants raciaux - excepté à l'antisémitisme racial. Grâce à une véritable « déconstruction-reconstruction ", ils usèrent du vocabulaire antisémite pour en détourner l'usage. Rejetant tout déterminisme racial fataliste et préférant le transformisme de Lamarck, ils expliquèrent les «faiblesses» mentales et (ou) physiques des juifs par les mauvaises conditions sociales et environnementales de la société moderne. Autrement dit, elles pouvaient être corrigées. Puisant alors dans l'hygiénisme allemand, ils prônèrent le

14 M. B. HART, Social Science (note 5), p. 1-15.

15 Ibid., p. 65-73.

16 Sur les statistiques en Allemagne, ainsi que le rapport que développa Ruppin avec l'usage des nombres, cf. M. B. HART, Social Science (note 5), p. 1-15, 7-60; J. M. Efron, Defenders of the Race (note 5), p. 166174; D. J. Penslar, Zionism and Technocracy (note 5), p. 80-93; Olivier BAISEZ, Architectes de Sion - La conception par les sionistes allemands de la colonisation juive en Palestine, Paris, Hermann, 2015, p. 43-57; E. Bloom, Arthur Ruppin (note 8), p. 70-75.

17 À noter que la statistique allemande fut quasi-indispensable pour ses travaux, car il puisa abondamment dans les données du Kaiserliche Statistische Amt.

18 À propos des idées raciales, eugéniques et antisémites en Allemagne et en Europe, cf. A. Pichот, $L a$ société pure (note 3), p. 307-354, 393-423; Dominique Aubert-Marson, Histoire de l'eugénisme: une idéologie scientifique et politique, Paris, Ellipses, p. 238-245; Paul WEINDLING, Hygiène raciale et eugénisme médical en Allemagne, 1870-1993, tome 1, Paris, La Découverte, 1998, p. 114-120, 129-130.

19 La plupart de ces idéologies ont des racines anciennes, datant parfois du XVII ${ }^{\mathrm{e}}$ siècle. Cependant, ce fut seulement à la fin du XIX ${ }^{\mathrm{e}}$ siècle que s'est opérée entre elles une fusion intellectuelle dont l'antisémitisme racial en fut l'un des courants les plus emblématiques. 
perfectionnement biologique, mental et institutionnalisé de la "race juive», grâce à plusieurs mesures socio-eugéniques (nous les aborderons en dernière partie). Ainsi, sociologie, biologie et État sont inextricablement liés ${ }^{(20)}$.

Néanmoins, Ruppin ne pouvait pas mettre en application ses théories dans un pays où une partie considérable des élites affichaient son antisémitisme. Comme d'autres juifs européens, le sociologue subit une phase de «transvaluation » ${ }^{(21)}$, passant de la haine de soi nourrie par l'antisémitisme ambiant à un nationalisme ardant, désireux de faire renaître l'honneur des anciens Hébreux en Palestine. Lorsqu'il quitta l'Allemagne, il transféra donc et appliqua ses legs intellectuels et idéologiques germaniques en Palestine, seule terre qui (selon lui) pourrait garantir la régénération de la race juive. Ruppin devint définitivement sioniste au cours des années 1900, cette voie étant la seule qui lui permettait de mener à bien ses projets. Son objectif: y promouvoir un sionisme pratique plus prononcé, c'est-à-dire (comme tous les sionistes) soutenir un projet de colonisation massive mais qui, contrairement au sionisme politique qui privilégiait dans un premier temps les négociations diplomatiques avec les Anglais et les Ottomans, prônait une installation immédiate et systématique en Palestine ${ }^{(22)}$. Sur place, Arthur Ruppin participa activement au développement des colonies et chercha à concilier au mieux ses théories raciales et socio-eugéniques avec la réalité du terrain ${ }^{(23)}$.

\section{Une bio-histoire de la « race juive»}

Pour définir la race juive, Ruppin mélangea histoire et biologie. Sa théorie a l'avantage de synthétiser travaux précédents et ajouts personnels, tout en respectant dans sa démarche l'aspect pluridisciplinaire si caractéristique du sionisme eugénique et racial allemand $^{(24)}$.

En mêlant théorie raciale et récit biblique, Arthur Ruppin chercha à proposer une identité juive servant le projet sioniste et à répondre aux théories antisémites - la principale étant que les juifs formeraient une race dégénérée. En citant abondamment les théories de ses pairs ${ }^{(25)}$, Ruppin défendit avec force la pureté - et par conséquent la respectabilité - de la race juive. Au fil des siècles et à n'importe quel endroit du globe, chaque juif aurait conservé trois éléments raciaux originels : araméen, bédouin et philistin ${ }^{(26)}$. Pour le prouver, Ruppin procéda à des comparaisons morphologiques entre des juifs représentés dans des œuvres d'art antiques et des photographies de juifs

20 A. Bloom, Arthur Ruppin (note 8), p. 52-55; J. M. Efron, Defenders of the Race (note 5), p. 3-12; M. B. HART, Social Science (note 5), p. 1-15.

21 L'expression est fréquemment utilisée par Etan Bloom dans son ouvrage consacré au sociologue.

22 A. Ruppin, Memoirs, Diaries, Letters (note 9), p. 75-79.

23 En ce qui concerne le rapprochement entre le sionisme et les sciences sociales et raciales, voir en particulier: J.M. Efron, Defenders of the Race (note 5), p. 166-174; E. Bloom, Arthur Ruppin (note 8), p. 70-75; M. B. HART, Social Science (note 5), p. 16-23, 61-66.

24 Nous nous basons désormais surtout sur The Jews of Today, Soziologie der Juden et The Jewish Fate and Future.

25 Tels que le philosophe Moses Hess ou J. M. Judt qui publia en 1902 Die Juden als Rasse, ou encore le généticien et sioniste Redcliffe Nathan Salaman.

26 A. Ruppin, Soziologie der Juden, vol. 1 (note 9), p. 15-18, 26-31, 33, 38; A. Ruppin, The Jewish Fate and Future (note 9), p. 19-20. 
contemporaines $^{(27)}$. Ces comparaisons pseudo-scientifiques l'amenèrent à affirmer que la morphologie juive resta inchangée au cours du temps et que, par conséquent, les juifs seraient un peuple-race biologiquement homogène, au sang pur et relativement préservé de tout métissage. Comment l'expliquer? Pour le sociologue, les pratiques eugéniques et endogamiques ancestrales des juifs auraient joué le rôle de garde-fous raciaux. Ainsi, les juifs du $\mathrm{XX}^{\mathrm{e}}$ siècle seraient les descendants directs des Hébreux de l'Ancien Testament, mais aussi les dépositaires du droit à habiter leur véritable Heimat: la Palestine.

Autre théorie à réfuter: les origines sémites des juifs. Avec les théories raciales, le terme «sémite» ne faisait plus seulement référence à une famille linguistique, mais aussi à un groupe racial, rassemblant de nombreux peuples-races - tels les Arabes ou les juifs - jugés inférieurs, contrairement aux races indo-européennes ${ }^{(28)}$. Mais dans les faits, le terme "antisémite» ciblait en particulier les juifs lorsque c'étaient des hommes politiques qui s'en servaient ${ }^{(29)}$. La méthode d'Arthur Ruppin fut d'user une nouvelle fois de l'iconographie pour extirper les juifs de cette théorie sémite ${ }^{(30)}$. Mieux: il arriva à affilier ces derniers à la race blanche - et par conséquent à les rapprocher racialement de leurs ennemis antisémites ${ }^{(31)}$.

Ainsi, par une relecture de l'histoire juive, l'usage de méthodes empiriques - comme l'emploi de représentations iconographiques - et des explications sociologiques l'endogamie des Hébreux - Ruppin arriva à dégager une vision cohérente de la «race juive», utile pour briser les théories antisémites et légitimer le projet sioniste. Cette vision s'est nourrie de toutes les composantes du sionisme racial (nationalisme, socioeugénisme, darwinisme social...) et résulte donc d'une construction intellectuelle complexe mais tout à fait déchiffrable par les historiens.

\section{Diagnostic et avenir de la «race juive»}

Ruppin a toujours assigné à sa vision bio-historique des fins pratiques ${ }^{(32)}$. Par son diagnostic sévère et alarmiste, le sociologue constatait la récente dégénérescence de la race juive, pourtant si talentueuse et jusqu'alors exemplaire en matière de pureté raciale. Pour ce sioniste convaincu, il fallait retrouver le glorieux fil de l'histoire juive et suivre les mesures eugéniques qu'il prescrivait. Encore une fois, la pluridisciplinarité du sionisme racial allemand est remarquablement respectée. En effet, pour Ruppin, si les Hébreux avaient préservé leurs caractéristiques raciales de l’assimilation, c’était

27 Les clichés en question, pris par Ruppin, se trouvent dans Soziologie der Juden (vol. 1), à la fin de l'ouvrage.

28 Autrement dit, si la construction nationale avait théorisé l'idée selon laquelle «un peuple = une langue», les théories raciales ont prolongé ce raisonnement en affirmant qu' « une langue = une race».

29 En ce qui concerne les termes «sémite», "antisémitisme» et «indo-européen», cf. Pierre-André Taguieff (dir.), Dictionnaire historique et critique du racisme, Paris, Presses universitaires de France, 2013, p. 92, 134, 885-887, 1484, 1665-1667.

30 A. Ruppin, The Jews of Today (note 9), p. 214; A. Ruppin, Soziologie der Juden, vol. 1 (note 9), p. 20 -21.

31 A. Ruppin, The Jews of Today (note 9), p. 213; A. Ruppin, Soziologie der Juden, vol. 1 (note 9), p. 18-19, 23-24.

32 Il ne s'agissait pas ici de détailler les réalités et les limites de la pratique ruppinienne sur le terrain mais la théorisation, en amont, de cette pratique. 
grâce à des mesures socio-économiques drastiques: interdiction des mariages mixtes, combat contre les dieux païens des autres peuples, monopole des activités commerciales, interdits alimentaires ${ }^{(33)}$... Depuis le XIX ${ }^{\mathrm{e}}$ siècle et l'affirmation en Occident des sociétés modernes, sécularisées et industrialisées, un retournement se produisait. Les juifs émancipés avaient davantage tendance à s'assimiler aux sociétés chrétiennes d'Europe de l'Ouest. Pour Arthur Ruppin, c'était une tragédie. Cette tendance entraînerait ce que le sioniste Max Nordau appelait la «dégénérescence» (Entartung) ${ }^{(34)}$. Les juifs, à présent assimilés, seraient en voie de dégénérescence. Leurs mauvaises habitudes sociales expliqueraient les «données statistiques» inquiétantes compilées par Ruppin sur les juifs d'Europe de l'Ouest ${ }^{(35)}$ : baisse de la natalité, de la masse musculaire, hausse de la mortinatalité, intellectualisme disproportionné... En d'autres termes, pour le sociologue, l’assimilation et le métissage étaient synonymes de déclin.

Néanmoins, Arthur Ruppin, apôtre du transformisme lamarckien, n’a jamais interprété ce déclin comme une fatalité. Tout pouvait encore être corrigé. Sa transformation intellectuelle - liée au phénomène de transvaluation - l'empêchait d'adhérer au déterminisme antisémite et le poussait, au contraire, à démontrer que les juifs demeuraient, encore et toujours, les fiers dépositaires de nombreux talents raciaux ancestraux, à la fois physiques et intellectuels. Ruppin renversa une dernière fois les arguments antisémites en faisant des juifs, supposés cupides et sans esprit créatif, un peuple qui donna au monde de nombreux génies - il citait entre autres Einstein, Marx, Freud... Tout en défendant (de manière nébuleuse) l'idée selon laquelle la race juive aurait apporté à la civilisation occidentale l'esprit rationnel, le sociologue cherchait à démontrer statistiquement la plus grande proportion de «génies» chez les juifs que chez tout autre peuple-race ${ }^{(36)}$. La « race juive» était pour lui une des plus remarquables et elle devait le rester.

Certes, il y avait un paradoxe chez Arthur Ruppin, qui développait une interprétation négative des effets de l'assimilation, tout en mettant en avant des juifs (du moins de son point de vue) célèbres mais assimilés (tels Einstein ou Freud). Mon hypothèse est que cette contradiction est symptomatique des théories et raisonnements animés par une idéologie extrême, voire extrémiste, et donc sans concession. L'enjeu n'est pas de déduire des résultats en partant d'un raisonnement logique et (ou) empirique, mais de faire en sorte que les différentes étapes de la démonstration amènent invariablement à un résultat tacitement postulé dès le départ. Dans ce cas de figure, la science ne décrypte pas le réel, mais apporte du crédit, une patine qui sera gage de véracité pour ce

33 A. Ruppin, The Jews of Today (note 9), p. 20, 24-25, 138-141; A. Ruppin, Soziologie der Juden, vol. 2 (note 9), p. 21-22.

34 M. B. Hart, Social Science (note 5), p. 125-127; Max Nordau, Dégénérescence, Paris, Max Milo Éditions, 2006, $182 \mathrm{p}$.

35 A. Ruppin, Soziologie der Juden, vol. 2 (note 9), p. 88-91. Étrangement, Ruppin ne source aucune de ces données statistiques. Cependant, même s'il s'agissait d'études statistiques réelles, on pourrait donner autant de crédit à ces études qu'à celles qui cherchaient à démontrer, via la craniométrie, l'infériorité des races «noire» et «jaune». Le racisme, le colonialisme et (ou) l'impérialisme y transparaissaient. Elles ne peuvent donc en rien se revendiquer de l'objectivité scientifique. Pour ces «scientifiques", il ne s'agissait pas d'observer le monde pour en déduire des lois, mais de prouver par tous les moyens des stéréotypes et des préjugés personnels.

36 A. Ruppin, The Jews of Today (note 9), p. 212, 215-216, 219-220; A. Ruppin, The Jewish Fate and Future (note 9), p. 23-24. 
qui est affirmé comme une réalité. Les courants racistes et nationalistes du XIX ${ }^{\mathrm{e}}$ siècle sont en cela d'excellents exemples. Cependant, une telle méthodologie, si éloignée des méthodes d'analyse modernes, engendre inévitablement des incohérences dans le raisonnement. Ruppin souhaitait à la fois critiquer l'assimilation et glorifier les grands juifs de l'histoire. Des incohérences étaient inévitables. Comme tous les raciologues, il ne voulait en aucun cas amender son système de pensée et ne prenait pas en compte les faits qui l'auraient modifié. Soit Ruppin n’a pas décelé cette incohérence (ce qui est peu probable car elle n'est de loin pas la seule présente dans ses ouvrages), soit il a préféré l'ignorer (ce qui nous paraît fort possible). Ruppin ne s'appuyait pas seulement sur son raisonnement, mais aussi sur des convictions; et même s'il cherchait à leur donner une valeur scientifique, ces dernières étaient davantage de l'ordre de la croyance. Dans ce cas, ce n'était pas quelques incohérences qui pouvaient ébranler un homme convaincu à l'avance de la pertinence de ses hypothèses.

Ainsi, pour Ruppin, la «race juive» était-elle donc tout à fait capable d'endiguer son déclin. Reprenant les théories du penseur allemand Ernst Haeckel, Ruppin était convaincu que la sélection naturelle et la transmission des caractères acquis régissaient l'évolution des races humaines. Le fatalisme racial n'existait donc pas. Pour lui, les juifs pouvaient se régénérer par cette sélection et cette transmission et même, en réinterprétant le troisième état du positivisme d'Auguste Comte, se dépasser soi-même grâce au pouvoir rationnel de la science. Ce dépassement engendrerait un nouvel homme juif, à l'image du surhomme nietzschéen, mais doué d'une dimension à la fois morale et physique ${ }^{(37)}$. Derrière cette idée de dépassement des limites biologico-sociales par la science, Ruppin comptait bien mettre en place un programme eugénique, fortement inspiré des hygiénistes allemands - mais relativement "modéré» en comparaison avec eux. La santé et l'hygiène des populations juives devaient être améliorées; les individus potentiellement dangereux ou trop faibles mis à l'écart ${ }^{(38)}$. L'autre mot d'ordre était l'entre-soi juif, de l'école aux activités économiques, en passant par la vie quotidienne. Ruppin considérait qu'un monde juif en vase clos était indispensable pour échapper au métissage et à la dégénérescence raciale, comme cela avait été le cas au contact des chrétiens d'Europe de l'Ouest ${ }^{(39)}$. Par-dessus tout, seul le retour en Palestine grâce au sionisme pouvait parachever cette renaissance. Ruppin adhérait aux théories des courants allemands völkisch qui supposaient un lien mystique, organique et immuable entre les hommes et la terre ${ }^{(40)}$. Pour se régénérer, les colons arrivant en Palestine devaient «imiter» les anciens Hébreux. Pour effacer leur faiblesse physique et leur intellectualisme, ils devaient s'adonner aux travaux agricoles, labourer la terre de leurs ancêtres,

37 E. Bloom, Arthur Ruppin (note 8), p. 48-50, 59-64.

38 A. Morris-Reich, «Arthur Ruppin's Concept of Race» (note 7), p. 7-8; Arthur Ruppin, "The Selection of the Fittest», Three Decades of Palestine: Speeches and Papers on the Upbuilding of the Jewish National Home, Tel-Aviv, 1936, p. 66-80. Cependant, Arthur Ruppin admettait dans ses écrits qu'une sélection mentale et physique des nouveaux arrivants en Palestine serait, dans la pratique, très difficile à mettre en œuvre. Par conséquent, il recommandait de mettre au moins à l'écart les individus porteurs de maladies infectieuses, ainsi que les malades mentaux et les épileptiques.

39 A. Ruppin, The Jews of Today (note 9), p. 238; D. J. Penslar, Zionism and Technocracy (note 5), p. 80-93.

40 E. Bloom, Arthur Ruppin (note 8), p. 55-57, 111-118; D. J. Penslar, Zionism and Technocracy (note 5), p. $80-93$. 
dans l'espoir de retrouver leur vigueur, leur force et leur virilité d'antan, et devenir les héritiers des Maccabées, ces juifs de l'Ancien Testament qui avaient combattu jusqu'à la mort pour défendre leur religion; celle-ci se confondant chez Ruppin avec leur race.

\section{Conclusion: vers un nouveau "paradoxe»?}

Analyser la notion de race juive sous la plume d'Arthur Ruppin nous a permis d'accéder à des pans entiers de la pensée raciale allemande et sioniste, de retrouver la richesse et l'originalité de ce courant idéologique - tout en le démystifiant, via une prudente déconstruction historique. Finalement, Arthur Ruppin et ses pairs différaient peu de leurs collègues raciologues allemands. Chacun d'entre eux était convaincu de l'existence de multiples races humaines, suivait le darwinisme social, croyait aux vertus de l'eugénisme, à la différence près que si les uns se délectaient des exploits de Siegfried, les autres ne juraient que par l'héroïsme des Maccabées.

Que reste-t-il aujourd'hui des théories d'Arthur Ruppin? La question mérite d'être posée en guise d'ouverture, même si elle nécessiterait de nouvelles recherches.

Certes, Arthur Ruppin, mort en 1943, demeure largement méconnu, même dans le milieu universitaire. Quant au monde d'après-guerre, il a marqué le terme «race» du sceau du national-socialisme et son antisémitisme, racial et annihilateur, n'est plus. Cependant, de temps à autre, certains faits actuels ressemblent à des spasmes, à des échos lointains, moribonds ou bien en perpétuelle évolution. La fin d'année 2017 fut en cela paradoxale. Le mercredi 6 décembre 2017, le président américain Donald Trump a reconnu unilatéralement la ville de Jérusalem comme capitale de l'État d'Israël. En réaction, des manifestations hostiles à cette démarche furent organisées en Europe. Ce fut par exemple le cas de l'Allemagne où, à Berlin, des drapeaux israéliens furent brûlés par des protestataires. Si le phénomène n'est pas nouveau, il inquiète ${ }^{(41)}$, à tel point que de nombreux politiciens songeraient à mettre en place un commissaire à l'antisémitisme en Allemagne ${ }^{(42)}$; tout un symbole. Néanmoins, au-delà de l'antisémitisme néonazi et des relents antisionistes (ou antisémites) chez certains migrants, fortement marqués dans leur pays d'origine par le conflit israélo-arabe, l'entrée tonitruante de l'AfD au Bundestag interroge ${ }^{(43)}$. Pour la première fois depuis l'après-guerre, un parti d'extrême droite s'impose avec force dans le paysage politique allemand. Organisation divisée en plusieurs courants, certains d'entre eux - les plus identitaires - demeurent ambigus en matière d'antisémitisme ${ }^{(44)}$.

41 Anne-Françoise Hivert, Thomas Wieder, «L'Europe craint un regain d'antisémitisme», Le Monde, 12 décembre 2017, http://www.lemonde.fr/international/article/2017/12/12/1-europe-craint-unregain-d-antisemitisme_5228503_3210.html.

42 Johanna Luyssen, "Allemagne: un commissaire à l'antisémitisme?», Libération, 19 décembre 2017, http://www.liberation.fr/planete/2017/12/19/allemagne-un-commissaire-a-1-antisemitisme_1617841. Notons également qu'entre 2016 et 2017 en Allemagne, les actes antisémites ont légèrement augmenté (6\%), dont $93 \%$ seraient liés à des mouvements d'extrême droite.

43 Le 24 septembre 2017, lors des élections fédérales allemandes, l'AfD (Alternative für Deutschland) fit un score de $13 \%$, ce qui lui permit de faire entrer plus de 90 députés au Bundestag.

44 Clément DAniez, "Législatives allemandes: "Il est absurde de comparer l'AfD aux nazis" ", L'Express, 25 septembre 2017, https://www.lexpress.fr/actualite/monde/europe/legislatives-allemandes-ilest-absurde-de-comparer-1-afd-aux-nazis_1946599.html; «En position de force, l'extrême droite 
Au même moment, alors que l'antisémitisme demeure vivace en Europe, des chercheurs israéliens tentent de démontrer que la judéité ashkénaze pourrait être déterminée par la génétique. Si les partisans de ces études mettent en avant la possibilité de trancher plus nettement la nature juive ou non-juive des nouveaux arrivants en Israël, d'autres s'inquiètent de voir la science prendre le pas sur l'identification de la judéité par la religion. Certains redoutent même qu'un usage systématique de la génétique par l'État hébreu - à la fois juif et démocratique - puisse engendrer une dérive eugénique de l'identité juive, ainsi qu'une discrimination entre ceux qui pourraient bénéficier des droits de citoyenneté et ceux qui en seraient exclus par la science ${ }^{(45)}$. Plus de cent ans après la publication de The Jews of Today et l'apogée de l'antisémitisme racial d'avantguerre, le faux «paradoxe» à la base de cette recherche semble bien persister au sein de nos sociétés occidentalisées. Arthur Ruppin n’a pas connu le même destin que d'autres théoriciens, plus illustres encore, des «sciences» raciales et du sionisme. Mais au-delà de ce constat, il a été représentatif d'une tendance intellectuelle de fond, à l'expansion et à la longévité bien plus importantes que ce que l'on aurait pu imaginer après 1945 . Si ses théories raciales sont aujourd'hui unanimement considérées comme erronées, on peut imaginer qu'il aurait accueilli avec enthousiasme ces nouvelles théories génétiques. Ainsi, de manière plus générale, on ne peut exclure qu'un usage dogmatique de la science du $\mathrm{XXI}^{\mathrm{e}}$ siècle pourrait amener l'ancienne terminologie raciale, soi-disant disparue, à faire sa mue; alors même que l'eugénisme et le racisme ne se sont jamais éteints. Ainsi, à l'autre antipode de notre paradoxe, si l'exacerbation de l'identité juive est toujours présente chez certains juifs, c'est également le cas de l'antisémitisme en Europe. Qu'adviendrait-il si un pouvoir extrémiste dirigeait un pays occidental et commencerait à s'intéresser à ces théories génétiques?

Arthur Ruppin est bien mort en 1943. Mais la notion de race juive, elle, n'a jamais vraiment disparu. Elle a simplement suivi les enseignements de Darwin, en s'adaptant à un nouvel environnement.

\section{Résumé}

Avant cet article, peu d'études francophones s'étaient intéressées aux sionistes allemands des XIX ${ }^{e}$ et $X X^{e}$ siècles qui affirmaient appartenir à une "race juive». Il s'agit ici de proposer une vision d'ensemble de ce sionisme racial, de mettre en relief son aspect pluridisciplinaire, ainsi que d'historiciser et de démystifier la notion de "race», encore sujette à moult polémiques. Pour y parvenir, une synthèse inédite des théories raciales d'un savant juif allemand majeur, le sioniste et sociologue Arthur Ruppin, a été entreprise. A

allemande tente de surmonter ses divisions", Le Monde, 2 décembre 2017, http://www.lemonde.fr/ europe/article/2017/12/02/en-position-de-force-1-extreme-droite-allemande-tente-de-surmonterses-divisions_5223747_3214.html; cf. Patrick Moreau, L'autre Allemagne: le réveil de l'extrême droite, Paris, Vendémiaire, 2017, 295 p.

45 Ilanit Chernick, "Juifs par l'ADN», The Jerusalem Post, 3 décembre 2017, http://www.jpost.com/ Edition-Francaise/Social-Eco/Juifs-par-1ADN-515872; en ce qui concerne l'histoire de ces études génétiques en Israël, cf. Nadia Aви EL-HaJ, The Genealogical Science. The Search for Jewish Origins and the Politics of Epistemology, Chicago/Londres, The University of Chicago Press, 2012; Shlomo SAND, Comment le peuple juiffut inventé. De la Bible au sionisme, Paris, Fayard, 2008, p. 379-388. 
travers la notion de "race juive» d'Arthur Ruppin, cet article aborde le poids écrasant des legs allemands (nationalisme exacerbé, usage des statistiques, eugénisme, darwinisme...) prêts à être mis en application en Palestine, mais aussi la vision unitaire de la "race juive» dans le temps et l'espace, ainsi que la peur maladive de sa dégénérescence et son obsessionnel souci de pureté.

\title{
Zusammenfassung
}

Vor diesem Artikel interessierte sich die französische Forschung kaum für die deutschen Zionisten des 19. und 20. Jahrhunderts, die glaubten, zu einer "jüdischen Rasse“ zu gehören. Ziel dieser Studie ist, einen Gesamtüberblick über diesen rassischen Zionismus zu vorschlagen, seinen interdisziplinäre Aussehen zu hervorheben sowie den noch umstrittenen Begriff der "Rasse" zu historisieren und zu entmystifizieren. Um dies zu erreichen, wurde eine gründliche Untersuchung der Rassentheorien des Zionisten und Soziologen Arthur Ruppin unternommen. Durch Ruppins Begriff der „jüdischer Rasse“ geht dieser Artikel das überwältigende Gewicht der deutschen Vermächtnisse (übersteigerter Nationalismus, Gebrauch von Statistiken, Eugenik, Darwinismus...) an, die in Palästina realisieren werden sollen, aber auch die einheitliche Vision der „jüdischen Rasse" in Zeit und Raum, sowie die kränkliche Angst ihrer Entartung und ihre zwanghafte Sorge um Reinheit.

\begin{abstract}
Before this article, few studies in French language ever cared about German Zionists of the $19^{\text {th }}$ and $20^{\text {th }}$ centuries who asserted to belong to a "Jewish race". The purpose here is to offer an overview of this racial Zionism, to emphasize its multidisciplinary aspect, as well as to historicize and demystify the notion of "race", still subject to many controversies. To do this, an unpublished synthesis of a major German Jewish scholar's racial theories, the Zionist and sociologist Arthur Ruppin, has been undertaken. Through Arthur Ruppin's notion of "Jewish race", this article addresses the overwhelming weight of German legacies (excessive nationalism, the use of statistics, eugenics, Darwinism...), ready to be implemented in Palestine, but also the unitary vision of "Jewish race" in space and time, as well as the unhealthy fear of its degeneration and its obsessional concern for purity.
\end{abstract}

